\title{
Hitching a ride with motor proteins
}

\author{
The biological machines that operate in cells are frequently a starting point for the development of \\ synthetic molecular motors.
}

Life is often a source of inspiration for researchers in nanotechnology. Adhesive surfaces have, for example, been created by using carbon nanotubes to mimic the microscopic hairs on the feet of geckos ${ }^{1}$, and novel photonic materials developed by understanding the intricate structure of butterfly wings ${ }^{2}$. In the field of molecular machines, this tactic is of particular relevance because there are already a variety of sophisticated ways in which to control molecular motion in nature.

Cells contain a range of biological motors that convert chemical energy into rotary and linear motion. ATP synthase, for example, is a rotary motor protein that is responsible for the synthesis of adenosine triphosphate (ATP), the universal fuel of a cell. Alternatively, proteins such as myosin, kinesin and dynein, are linear motors that travel along polarized cytoskeletal filaments powered by the hydrolysis of ATP. Myosins move along actin filaments and are involved in activities such as muscle contraction; kinesin and dynein walk along microtubules, transporting cargoes within the cells. Inspired by these examples, researchers have sought to create new machines and capabilities, and the range of current possibilities is illustrated in a number of articles in this issue of Nature Nanotechnology.

On page 33, Zev Bryant and colleagues show that myosins can be engineered to travel over longer distances and at higher speeds than their natural counterparts. Myosins typically have two motor domains (or heads), but these modified motors have three or four heads, as well as a flexible element between them. This allows the motors to travel along the filament tracks at up to $10 \mu \mathrm{m} \mathrm{s}^{-1}$; processive motion in natural myosins has previously been recorded at speeds of up to $7 \mu \mathrm{m} \mathrm{s}^{-1}$ (ref. 3).

Elsewhere, on page 44, Andrew Turberfield and colleagues show that DNA can be used to add an element of control to motor proteins. In cells, kinesins are known to be involved in the organization of microtubule networks. By attaching reconfigurable DNA nanostructures to such proteins, the team show that sequences of DNA can be used to direct the assembly of similar arrays of microtubules. Furthermore, the DNA-kinesin hybrids can transport

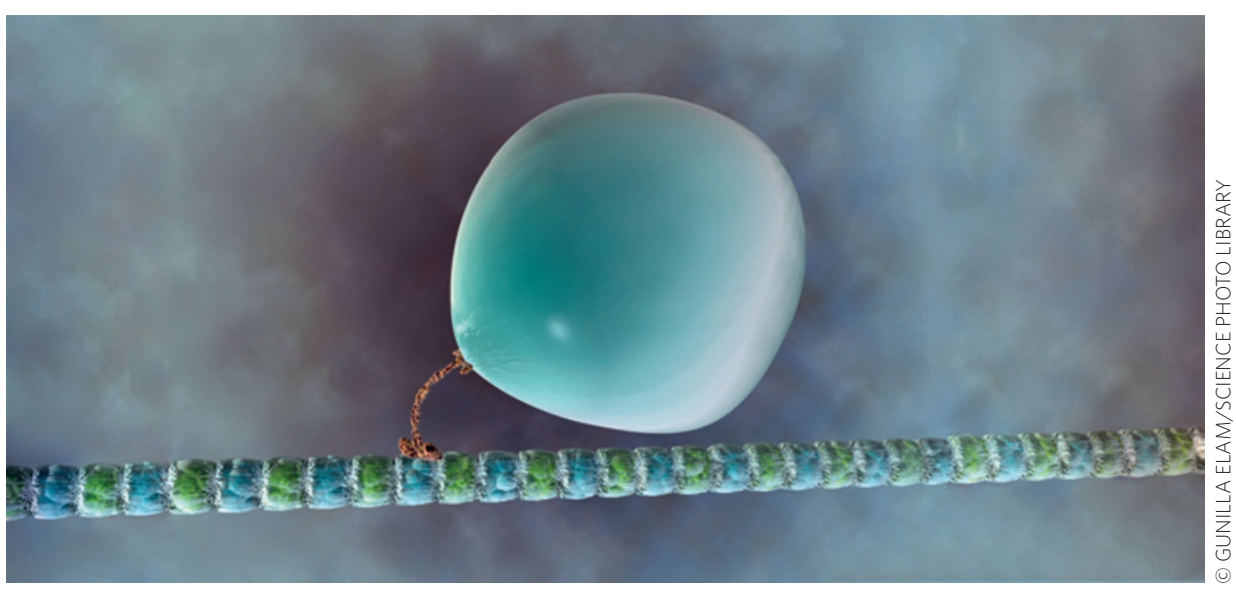

molecular cargo to the centre of the arrays and send signals to release the cargo or even disassemble the network of tracks.

DNA is often the molecule of choice for creating static self-assembled nanostructures. (See, for example, page 74, where Tim Liedl and colleagues have used DNA origami - a self-assembly technique in which a long single strand of DNA is folded into a predetermined shape with the help of short 'stapling' strands - to organize different nanomaterials into functional structures.) And it is proving to be of similar value in the fabrication of nanomachines. However, rather than being used to modify motor proteins, DNA is typically used to create 'DNA walkers' - motors made of DNA that run along self-assembled tracks that are also made of DNA - and the programmability of the molecule has allowed, for example, nanoscale assembly lines to be fabriacted ${ }^{4}$.

On page 39, Jong Hyun Choi and colleagues show that DNA walkers can also operate on tracks made of single-walled carbon nanotubes. The nanotube tracks are coated with RNA molecules, which the motor - a DNA enzyme - uses to fuel its steps along the one-dimensional track. The processive and autonomous motion along the track is similar to that of the motor proteins found in cells, and the motors can also, like motor proteins, carry cargo (cadmium sulphide nanoparticles). However, whereas proteins such as kinesin and dynein can take forward and backward steps along their tracks, the DNA motor destroys its track when it walks and can, therefore, only step in one direction.

Choi and colleagues also show that movement of the motor along the tracks can be imaged in real time with the help of the visible fluorescence of the nanoparticle cargo and the near-infrared emission of the nanotube track. This allows the team to carefully analyse the kinetics of individual motors, and they find that the motors can walk several micrometres and travel at speeds of up to around $0.1 \mathrm{~nm} \mathrm{~s}^{-1}$. Like other DNA motors ${ }^{5}$, these motors are much slower than motor proteins (and, moreover, the modified motors of Bryant and colleagues). However, as is the case with many DNA nanotechnologies, the level of control is impressive, and Choi and colleagues are able to start and stop the motion of the motors remotely by altering their chemical environment.

As these three papers show, in attempts to recreate and advance the capabilities of intracellular motors like myosin, kinesin and dynein, researchers are exploring a variety of approaches, from modifying biological motors, to creating natural-synthetic hybrids, to fabricating purely synthetic structures. The result is increasingly sophisticated ways in which to control molecular motion in the laboratory.

\footnotetext{
References

1. Qu, L., Dai, L., Stone, M., Xia, Z. \& Wang, Z. L. Science 322, 238-242 (2008)

2. Kolle, M. et al. Nature Nanotech. 5, 511-515 (2010).

3. Tominaga, M. et al. EMBO J. 22, 1263-1272 (2003).

4. Gu, H. Z., Chao, J., Xiao, S. J. \& Seeman, N. C. Nature 465, 202-205 (2010).

5. Wickham, S. F. J. et al. Nature Nanotech. 6, 166-169 (2011).
} 